\title{
Reflets
}

Revue ontaroise d'intervention sociale et communautaire

\section{Dyane Adam (dir.), Femmes francophones et pluralisme en milieu minoritaire, Ottawa, les Presses de l'Université d'Ottawa, 1996.}

\section{Denyse Côté}

Volume 3, numéro 2, automne 1997

Visibles et Partenaires : Pratiques et recherches féministes

URI : https://id.erudit.org/iderudit/026191ar

DOI : https://doi.org/10.7202/026191ar

Aller au sommaire du numéro

Éditeur(s)

Reflets : Revue ontaroise d'intervention sociale et communautaire

ISSN

1203-4576 (imprimé)

1712-8498 (numérique)

Découvrir la revue

Citer ce compte rendu

Côté, D. (1997). Compte rendu de [Dyane Adam (dir.), Femmes francophones et pluralisme en milieu minoritaire, Ottawa, les Presses de l'Université d'Ottawa, 1996.] Reflets, 3(2), 306-311. https://doi.org/10.7202/026191ar

Tous droits réservés (C) Reflets : Revue ontaroise d'intervention sociale et communautaire, 1997
Ce document est protégé par la loi sur le droit d'auteur. L'utilisation des services d’Érudit (y compris la reproduction) est assujettie à sa politique d'utilisation que vous pouvez consulter en ligne.

https://apropos.erudit.org/fr/usagers/politique-dutilisation/ 


\section{Femmes francophones et pluralisme en milieu minoritaire}

sous la direction de Dyane Adam, Ottawa, les Presses de l'Université d'Ottawa, 1996

par

Denyse Côté, Université du Québec à Hull

La thématique qui traverse l'ensemble de ce recueil est celle de la construction identitaire des femmes francophones de l'Ontario en rapport avec le pluralisme de leurs situations et de leurs identités. Il collige certaines présentations faites lors du Deuxième colloque du Réseau des chercheures féministes de l'Ontario français qui s'est tenu à l'Institut d'études pédagogiques de l'Ontario les 3, 4 et 5 mars 1995. Fidèle à ce qui est en voie de devenir une tradition, cette rencontre a été l'occasion d'un maillage de militantes, d'intervenantes, de bénévoles, de professionnelles et de chercheures féministes, en somme un moment privilégié de mise en commun de travaux et de réflexions sur les femmes francophones en milieu minoritaire. Bien qu'il soit basé en Ontario et qu'il réunisse une majorité d'Ontariennes et de travaux portant sur l'Ontario, nous y retrouvons également des analyses sur les Fransaskoises, les Acadiennes et les Québécoises. Ces textes appartiennent à différents registres : témoignages, analyses de pratiques et d'interventions, recherches fondamentales et recherches-actions. C'est là toute la richesse de cette entreprise.

Produire un tout cohérent à partir d'une matière première relativement éclectique n'est pas une mince tâche. Mission accomplie avec brio par le biais d'une double ordonnance : selon la nature du texte (recherche, analyse de pratiques, témoignages) et selon les thèmes abordés (construction identitaire, formation/ 
éducation, santé/bien-être personnel).Les douze textes du recueil sont variés et riches en perspectives, car les intérêts des chercheures, leurs appartenances disciplinaires respectives, leurs objets de recherche ainsi que leurs questionnements très différents convergent de façon harmonieuse autour du thème retenu. Comment vous les rapporter sans tronquer la richesse des analyses et sans vous présenter une liste d'épicerie de ces travaux? J'ai choisi de résumer chaque article au risque - j'en conviens - d'élaguer la richesse des réflexions mais dans le but de permettre, je l'espère, de saisir la trame de chaque texte et de donner l'envie d'y voir plus loin.

\section{Construction identitaire}

Les questions posées par Natalie Beausoleil sont les suivantes: comment les minoritaires se définissent-elles et quelles sont les limites de la conceptualisation des théoriciennes féministes blanches en regard des stratégies de résistance et de définition identitaire des femmes noires et latino-américaines? Le rapport de la chercheure blanche aux femmes des minorités visibles comme sujets de recherche, la nature changeante de l'identité, la question des identités multiples, la signification variée des identités selon les individus et les groupes, la négociation constante de l'identité et de l'altérité imposée de l'extérieur par le groupe majoritaire en rapport avec les luttes pour l'espace politique sont tour à tour abordés. À la lumière de ces questions, elle reprend certains éléments de sa recherche sur les pratiques d'apparence des femmes.

Christiane Bernier présente une riche analyse des discours sur l'identité en Ontario français. Elle aborde plus précisément la construction du discours minoritaire franco-ontarien à partir de l'exclusion des autres ethniques francophones et du rapport contradictoire entre ces autres francophones et les francophones d'origine qui reproduit une certaine logique de domination et qui reflète la limite théorique des lieux où est pensée l'identité des minoritaires (p. 23). Elle souligne également comment le discours francoontarien ne permet pas l'intégration d'autres facteurs de discrimination que ceux qu'il reconnaît lui-même comme constitutifs de son rapport au majoritaire et qui sont corrélatifs à l'historicité de sa 
communauté telle qu'il la construit (p. 22). L'exclusion des discours sur le genre illustrent bien ce mécanisme.

Caroline Andrew et Mythili Rajiva présentent les processus de la légitimation des acteurs comme partie prenante des enjeux d'un système politique car cette légitimation change la nature $d u$ débat et les politiques publiques qui en résultent (p. 32). Elles tentent donc de saisir la construction d'une identité pour les femmes francophones d'Ottawa appartenant à une minorité visible à travers l'analyse des objectifs concrets de trois programmes municipaux: la politique des services en français, la politique sur le multiculturalisme et les politiques touchant les femmes. Leur évaluation est mitigée pour plusieurs raisons, notamment parce que ces politiques ne rejoignent pas les femmes à titre de groupe ou de communauté, mais uniquement à titre individuel.

Isabelle McKee-Allain analyse la question du pluralisme religieux dans les écoles francophones du Nouveau-Brunswick. Selon McKee-Allain, il s'agit là d'un dossier révélateur de tendances sociales globales et qui pose, entre autres, les enjeux de la production de l'ethnicité à l'intérieur du réseau institutionnel de l'éducation traversé de multiples contradictions et ambiguïtés. Ces enjeux sont d'autant plus intéressants que c'est une minorité acadienne appartenant à certaines églises protestantes fondamentalistes qui pose la question du pluralisme religieux en milieu scolaire et, par extension, celle du rôle de la religion catholique dans la définition de l'identité acadienne à l'intérieur des institutions provinciales.

\section{Formation et l'éducation}

Marie-Josée Berger pose la question de la visibilité réelle des femmes francophones appartenant à des minorités visibles. Même si les documents gouvernementaux décrivent la société ontarienne comme étant pluraliste, ce plurarisme n'a pas encore été défini comme étant englobant et essentiel au développement de la société ontarienne (p. 55). Ainsi, le fossé est grand entre les principes énoncés et l'implantation des politiques. Selon Berger, l'absence de définitions du pluralisme social ontarien est un obstacle de plus à l'insertion 
des femmes francophones de minorités visibles qui doivent s'insérer dans un marché du travail où l'anglais est la langue dominante, qui font face à une déqualification à leur arrivée au pays, à la difficulté de s'insérer dans les programmes de formation offerts, aux limites que leur statut d'immigration leur impose et enfin à la discrimination relative à leur couleur et à leurs responsabilités familiales.

Denise Lemire présente une étude sur les obstacles à la formation chez les femmes francophones en regard des programmes du Conseil ontarien de formation et d'adaptation de la main-d'œuvre (COFAM) - aboli depuis par le gouvernement conservateur. Elle pose le retour aux études comme problématique sociale qui doit se définir en rapport avec le marché du travail, mais aussi en interface avec les désir des femmes-utilisatrices de poursuivre leur formation. Pour ceci, il faudrait, entre autres, que les services de counselling et les programmes de formation soient offerts en français et que des accommodements soient faits pour la situation particulière des femmes (besoin de services de garde, accueil du milieu du travail pour les femmes, etc.).

\section{Santé et le bien-être personnel}

Jacinthe Michaud analyse les mécanismes ayant permis au mouvement féministe sur la santé des femmes de se tailler un espace dans l'arène politique québécoise. L'auteure passe en revue différents aspects de la formation d'un contre-discours en santé des femmes, du potentiel de représentation discursive du mouvement des femmes en santé, des dichotomies qui le traversent, des fondements du contre-discours ainsi que différents positionnements stratégiques en regard de l'hégémonie interne de ces discours qui refusent une représentation adéquate de la diversité des femmes. Par le biais de cette analyse, elle appelle à la mise sur pied, en Ontario, d'une collective francophone qui tienne compte de l'expérience des femmes francophones en matière de santé.

Anne-Marie Ambert et Louise D. Gagnon ont cherché à connaitre l'effet de la délinquance des adolescent sur les parents. Les mères des garçons semblent plus affectées négativement que les pères en 
général et que les mères des filles retenues aux fins de cette étude. Ces résultats corroborent le fait que les mères s'occupent de façon plus soutenue des enfants et prennent souvent en charge la médiation avec les institutions sociales et juridiques. Cependant, les auteures croient que ces résultats sont aussi liés à la gravité des gestes délinquants commis et au fait que les adolescents ou adolescentes ayant commis des gestes plus graves sont aussi souvent plus incontrôlables à la maison. La prostitution, la fugue et l'itinérance étant les comportements jugés les plus graves habituellement imputés aux filles, les chercheures n'ont pu vérifier cette hypothèse puisque ces comportements ne relèvent pas de la Loi des jeunes contrevenants, laquelle a servi de point de départ pour l'échantillonnage.

Manon Lemonde et Johanne Pomerleau présentent l'impact d'un programme d'entraide auprès des personnes âgées, en particulier auprès de femmes francophones. Pour maintenir un état de bienêtre, les personnes âgées doivent réaliser un équilibre entre les périodes de solitude et d'interaction sociale. Le programme Réseau d'entraide, dont les auteures font l'analyse, a pour but de donner aux personnes âgées les outils nécessaires pour maintenir leur qualité de vie. Les auteures identifient les apprentissages faits par les femmes francophones dans ce contexte et le succès de la démarche tel que mesuré par les participantes.

\section{Témoignages}

Jocelyne J. Paquette décrit l'isolement des femmes francophones de Thunder Bay. Celles-ci vivent dans un milieu hostile au français et aux francophones et doivent, à titre d'exemple, franchir 2000 $\mathrm{km}$ (la distance les séparant de Toronto) pour assister à des conférences ou des réunions en français. Elle décrit sa propre construction identitaire comme francophone symbolique par des membres de la majorité anglophone, processus qui empêche toute inclusion, acceptation ou réel respect des diversités. Elle souligne, d'autre part, le fait que de nombreuses Franco-Ontariennes considèrent le féminisme comme une anomalie. 
Françoise Singur-Cloutier fait état d'une recherche-action menée auprès et avec des Fransaskoises dans le but de construire une base d'unité solide malgré les distances, les divisions entre le rural et l'urbain, et les différences de statuts et de valeurs. Cette recherche visait à connaître le vécu et les perceptions des Fransaskoises afin d'avoir une meilleure compréhension de leurs besoins, de leurs intérêts et de leurs motivations. Une des conclusions de cette étude révèle la portée paradoxale du bénévolat, à la fois nécessaire à la survie de la communauté et objet de valorisation et de soutien pour les femmes mais aussi source importante de travail pour celles-ci.

Sylvie D'Augerot-Arend pose la question des obstacles que confrontent les Francophones immigrantes indépendantes. Ils sont multiples et vont de la perception tronquée du pays d'origine de l'immigrante (en particulier la perception des hommes anglophones en regard des femmes françaises), de l'hostilité ou de la méfiance en rapport avec les caractères physiques de l'immigrante ou de certains de ses comportements (garder son nom, son apparence et surtout opter pour l'intégration à la minorité francophone). Le fait que l'immigrante soit indépendante dérange aussi. L'auteure décrit également les mécanismes de favoritisme négatif dont elle a été victime à l'intérieur des universités.

Bref, ce livre est incontournable si vous vous intéressez à la question de l'identité en milieu minoritaire et si vous vous intéressez à la question du pluralisme en regard du féminisme et de la francophonie. Nous attendons donc avec impatience les Actes du Troisième colloque du Réseau des chercheures féministes de l'Ontario français tenu à Sudbury en mai 1997. 